\title{
Masking of obstructive sleep apnoea by drug induced central sleep apnoea
}

\begin{abstract}
Benzodiazepines are widely prescribed hypnotic agents which have multiple proven neurological and respiratory side effects. However, literature is sparse with regards to the incidence and occurrence of new onset central sleep apnoea in individuals being treated with benzodiazepines for insomnia. We present a case report of a patient presenting with new onset central sleep apnoea secondary to long term usage of benzodiazepines, with resultant masking of his pre-existing obstructive sleep apnoea.
\end{abstract}

Key words: central sleep apnoea, benzodiazepines, obstructive sleep apnea

Adv Respir Med. 2019; 87: 189-193

\section{Introduction}

Benzodiazepines are the most widely prescribed hypnotic agents for the short-term management of insomnia [1]. These agents normally exert their influence by decreasing the sleep latency and prolongation of total sleep time [2], however, they worsen sleep quality by decreasing REM and N3 stages of sleep. Other possible side effects include tolerance to the hypnotic effects [3], rebound insomnia upon abrupt discontinuation, reduced slow wave sleep $[4,5]$ and withdrawal effects. However, literature with regard to the incidence and occurrence of new onset central sleep apnoea (CSA) in individuals being treated with benzodiazepines for insomnia is limited to case reports with sparse texts on the pathophysiology behind it. We present a case of a patient with new onset central sleep apnoea secondary to long-term usage of benzodiazepines, with resultant masking of his pre-existing obstructive sleep apnoea (OSA).

\section{Material and methods}

A 53-year-old male, with no comorbid illness in the past, presented to the department of pulmonary, critical care and sleep medicine with complaints of snoring, excessive daytime somno- lence, mild cognitive impairment and nocturia for a period of 1 year. He had a past history of usage of tablet alprazolam $0.5 \mathrm{mg}$ at night time, which was prescribed by a medical practitioner for treatment of insomnia for the past six months. The patient had an Epworth sleepiness score (ESS) of 15. His body mass index (BMI) was 22. The man was a non-smoker. On evaluation, his pulmonary function test and echocardiography were within normal limits. There was no history of any cardiovascular or cerebrovascular event.

The man underwent a diagnostic polysomngraphy (PSG) Level 1 which recorded the presence of central apnoeic events (Figure 1, 2). He had an apnoea-hypopnoea index (AHI) of 20 with more than fifty percent of events being central, hence a diagnosis of CSA was made in accordance with the American Academy of Sleep Medicine (AASM) guidelines [6].

The PSG was scored manually by a sleep physician. In consultation with a psychiatrist, alprazolam was discontinued and the patient was initiated on non-benzodiazepine medication (zolpidem $5 \mathrm{mg}$ once a day). The magnetic resonance imaging (MRI) of the brain was performed to evaluate CSA associated with neurologic disease; it was reported normal. On subsequent evaluation after 2 weeks, the patient reported a subjective mild

Address for correspondence: Pranav Ish, Department of Pulmonary, Critical Care and Sleep Medicine, VMMC and Safdarjung Hospital, New Delhi, India;

e-mail: pranavish2512@gmail.com

DOI: 10.5603/ARM.2019.0030

Received: 13.12.2019

Copyright (C) 2019 PTChP

ISSN 2451-4934 


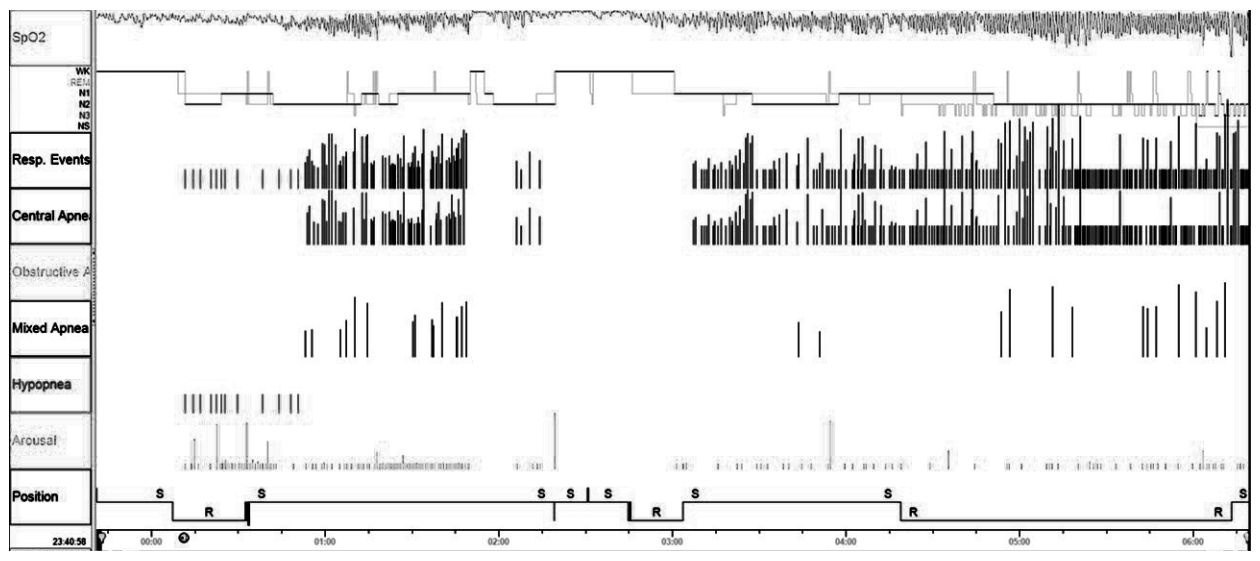

$\mathrm{SpO}_{2}$ — oxygen saturation; WK — wake; REM — rapid eye movement sleep; Resp events — respiratory events; $\mathrm{S}$ — supine; $\mathrm{R}$ — right lateral

Figure 1. Hypnogram showing numerous central sleep apnoeas and desaturations and poor quality sleep (most time spent in N2 with no REM sleep)

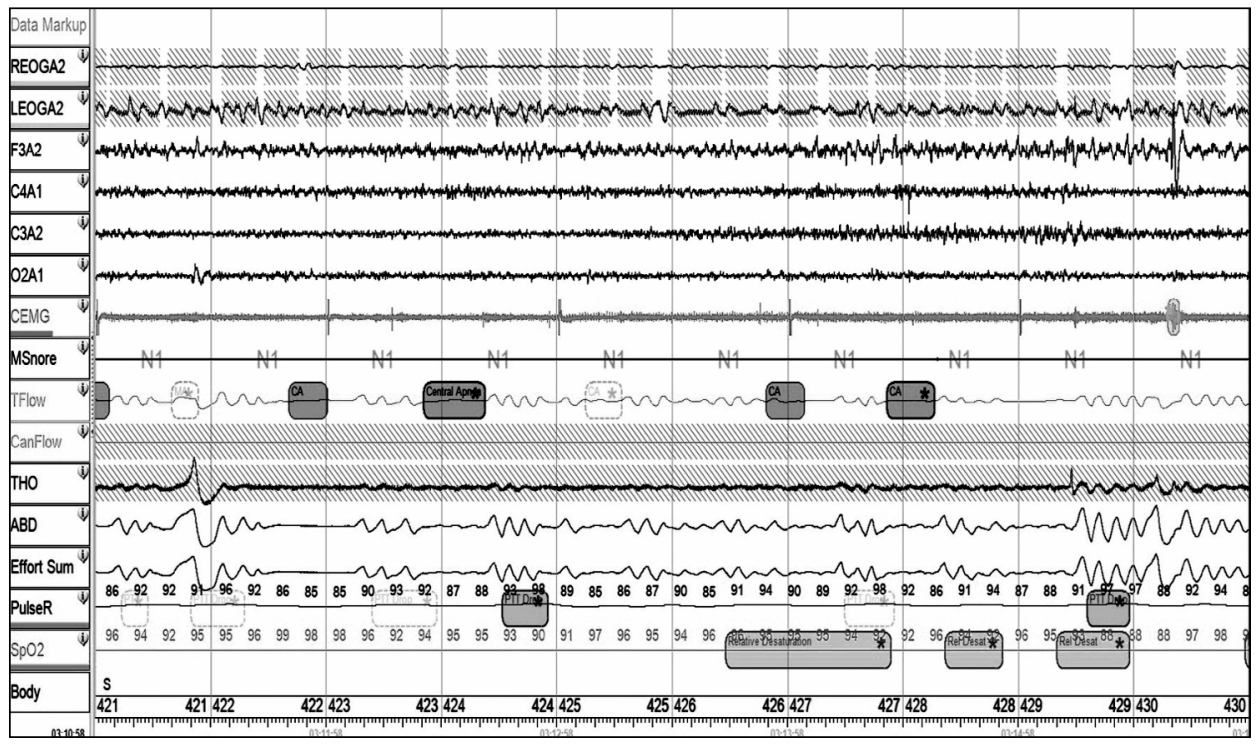

REOGA2 and LEOGA2 - right and left electrooculography; F3A2, C4A1, C3A2 and 02A1 - electroencephalograph; CEMG - Chin electromyography; mSnore - snoring; T flow — thermistor flow; Can flow — nasal cannula flow; THO — thoracic muscle effort; ABD — abdominal muscle effort; Effort sum — thorax and abdominal effort sum; PulseR — pulse rate; $\mathrm{SPO}_{2}$ — oxygen saturation; Body — body position

Figure 2. Polysomnography five-minute window with epoch 421-430 showing central sleep apnoea in view of no effort with no flow

improvement in sleep quality with ESS getting better to 11 from an initial value of 15 over a period of two weeks. The effect of alprazolam wears off in 2 to 3 days as it has a half-life of around 11 hours.

However, in view of persistent daytime somnolence, a repeated PSG was planned. Unexpectedly, in the PSG, numerous obstructive hypopnoeas were recorded with an AHI of 32 (Figure 3-5). There was a complete absence of central apnoeas and hypopnoeas. The present study did not use the esophageal pressure measurements; differentiation between obstructive and central hypopnoea was performed manually. In general, central hypopnoeas are defined by a proportiona- te decrease in both airflow and respiratory effort. Usually, there is no snoring, absence of chest-abdominal paradox and the nasal pressure or PAP device flow signal is fairly rounded.

Split night titration was done and a continuous positive airway pressure (CPAP) of $5 \mathrm{~cm}$ water was sufficient to abolish all the obstructive apnoea and hypopnoea events, however, few hypopnoeas persisted in supine REM sleep leading to a "good titration” according to the AASM guidelines [6]. The sleep quality improved with supine REM achieved after continuous positive airway pressure (CPAP) titration (Figure 6,7 ). The patient was continued on tablet zolpidem for one 


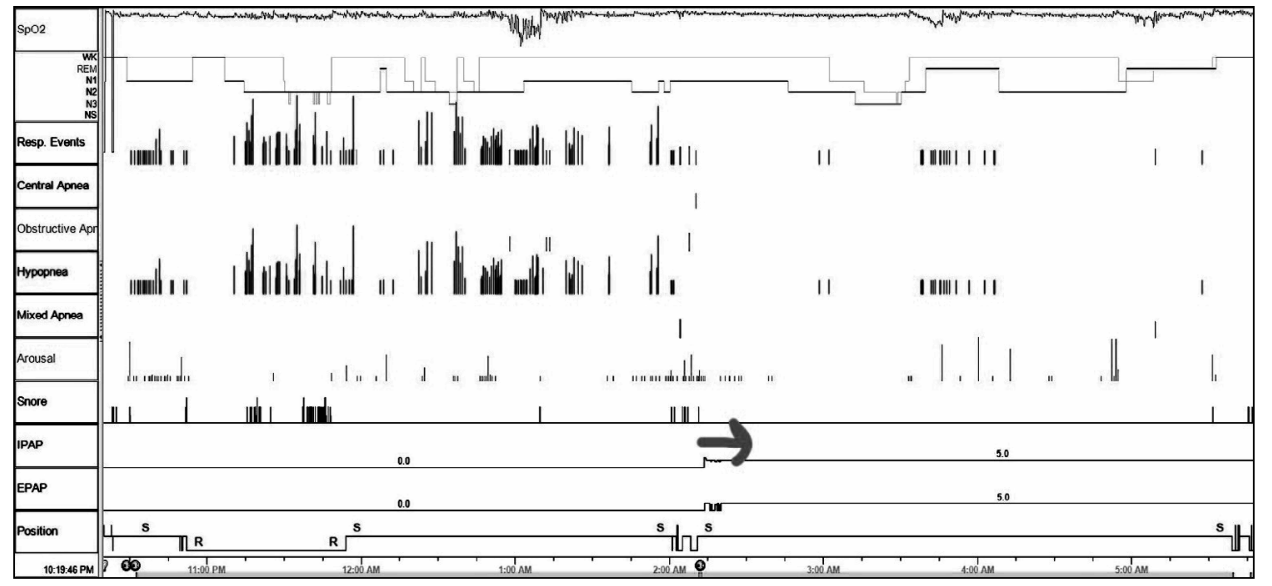

$\mathrm{SpO}_{2}$ - saturation of oxygen; WK - wake; REM - REM sleep; $\mathrm{S}$ - supine; $\mathrm{R}$ - right lateral; IPAP — inspiratory positive airway pressure; EPAP - expiratory positive airway pressure

Figure 3. Hypnogram showing disappearance of central events and appearance of obstructive hypopneas in follow-up polysomnography which is titrated with CPAP (green arrow) achieving supine REM

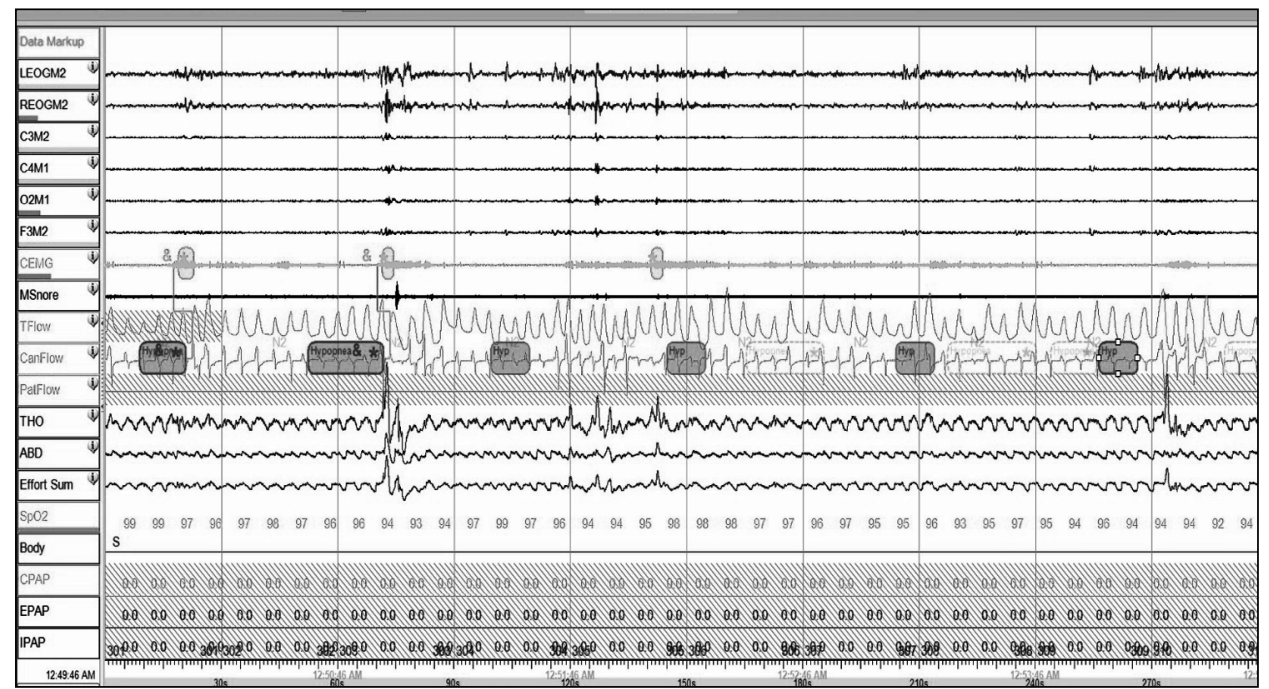

REOGM2 and LEOGM2 — right and left electrooculographyl F3M2, C4M1, C3M2 and 02M1 - electroencephalograph; CEMG — Chin electromyography; mSnore - snoring; T flow - thermistor flow; Can flow — nasal cannula flow; PatFlow — Patient flow using positive airway pressure device; THO — thoracic muscle effort; $\mathrm{ABD}$ — abdominal muscle effort; Effort sum — thorax and abdominal effort sum; PulseR — pulse rate; SPO2 — oxygen saturation; Body — body position; IPAP — inspiratory positive airway pressure; EPAP — expiratory positive airway pressure

Figure 4. Polysomnography with ten epochs number 301-310 showing numerous obstructive hypopnoeas

more week and prescribed a continuous airway pressure of $6 \mathrm{~cm}$ water for nocturnal use. The man is currently on follow-up on CPAP only with no sedative, and reports an improved sleep quality and reduced daytime symptoms. The Epworth sleepiness score is now 5 with no limitation in social and workplace activity.

\section{Discussion}

Usage of hypnotics for sleep induction and maintenance is the most common short-term management strategy for insomnia. However, benzo- diazepines have been implicated in precipitating sleep apnoea, especially central sleep apnoea (CSA) in patients with heart failure and severe desaturations in patients with mild OSA [7].

This situation is complicated by many factors, misdiagnosis of OSA as insomnia, ease of access to certain sedatives and comorbid psychiatric diseases. The other contributing factors are lack of awareness on the part of the physician to recognise the symptoms of choking, snoring, early morning headache suggestive of OSA and worsening of OSA by psychiatric medications. Moreover, if an airway obstruction occurs, aro- 


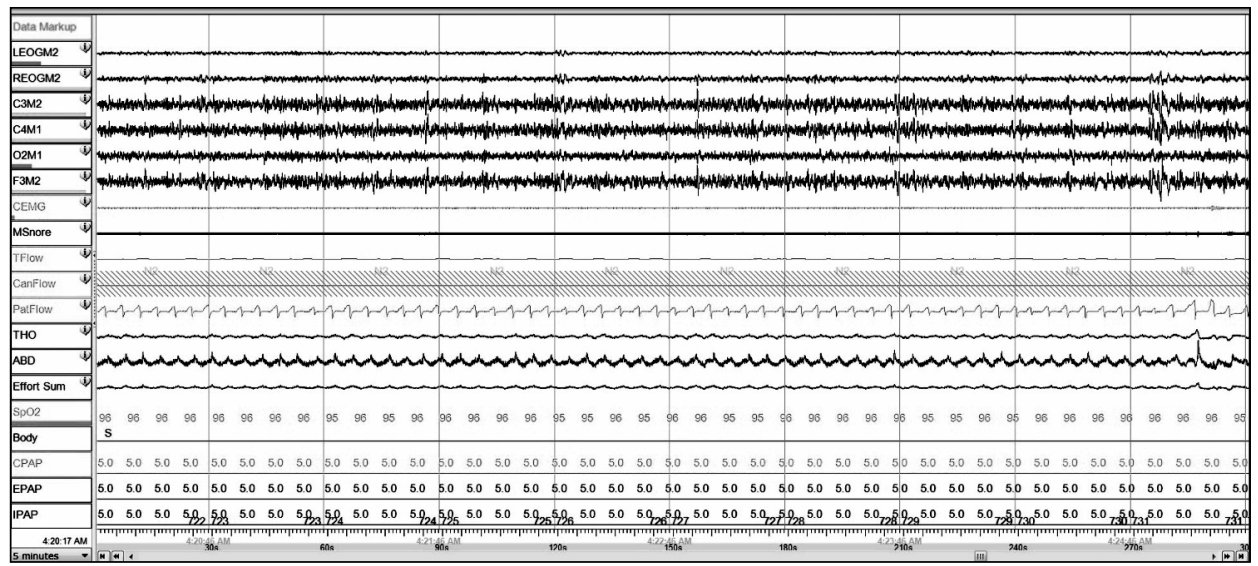

REOGM $_{2}$ and LEOGM2 - right and left electrooculography; F3M2, C4M1, C3M2 and 02M1 - electroencephalograph; CEMG - Chin electromyography; mSnore - snoring; T flow — thermistor flow; Can flow — nasal cannula flow; PatFlow — Patient flow using positive airway pressure device; THO — thoracic muscle effort; ABD — abdominal muscle effort; Effort sum — thorax and abdominal effort sum; PulseR — pulse rate; SP02 - oxygen saturation; Body — body position; IPAP — inspiratory positive airway pressure; EPAP — expiratory positive airway pressure

Figure 5. Polysomnography with ten epochs number 721-731 showing no residual hypopnoeas on positive airway pressure of $5 \mathrm{~cm}$ water



WK — wake; REM — rapid eye movement sleep

Figure 6. No REM and minimal N3 stage activity recorded in initial polysomnography when patient was taking Alprazolam

usals allow the patient to correct the apnoea. Benzodiazepines can prevent arousal by causing sedation, thereby worsening the obstruction and associated desaturation. However, another school of thought reasons that as OSA worsens in REM sleep, benzodiazepines by decreasing REM sleep and allowing for breathing pattern to stabilise, prevent OSA to manifest. Even though literature is sparse to support either hypothesis, a combination of the above mechanisms may nullify each other as shown in a Cochrane database demonstrating no worsening of AHI by sedative drugs. Alprazolam was not directly evaluated, however, eszopiclone, zolpidem, flurazepam, temazepam, nitrazepam, triazolam, sodium oxybate and Ramelteon were included in the studies of the database [8].

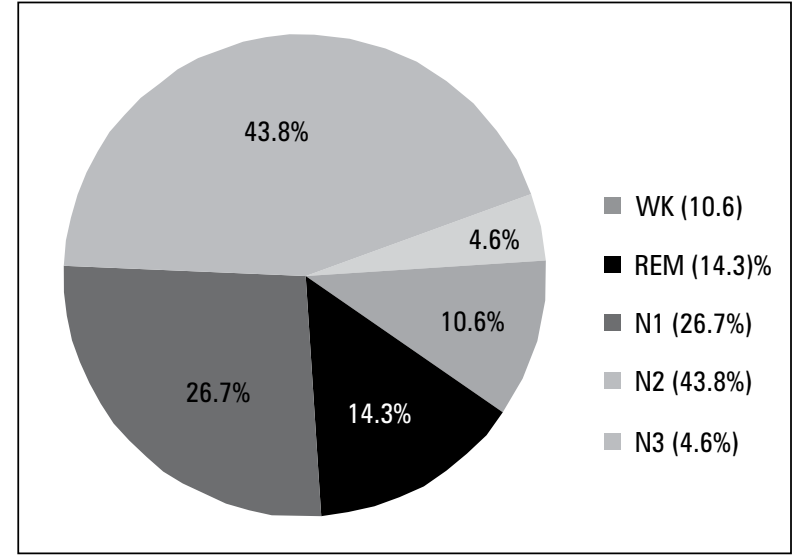

WK — wake; REM — rapid eye movement sleep

Figure 7. Improvement in sleep quality with REM contributing to $14 \%$ of sleep after stopping alprazolam and on PAP titration

The occurrence of CSA has been reported by benzodiazepines because as central nervous system depressants, these drugs decrease the central respiratory drive which also has been challenged by authors claiming that hypnotics not only improve sleep but also decrease apnoea frequency probably by reducing arousals and elevating arterial $\mathrm{pCO}_{2}$ [9]. However, masking of OSA as CSA has not been reported in literature. Muscle relaxants like baclofen have been reported to cause CSA [10], thus giving a plausible reason to benzodiazepines to mask OSA as CSA by their weak myo-relaxant action.

Esophageal pressure measurement was not carried out for differentiating between central and obstructive events in our patient in view of lack of consent for the same. Hence, the differentiation 
between obstructive and central hypopnoea was performed manually — rather visually. The AASM guidelines recommend esophageal pressure and respiratory inductance plethysmography (RIP) belts as gold standard for effort and RIP belts were used in our case. The AASM Task force members recognise that a decrease in RIP excursions cannot differentiate obstructive and central hypopnoeas because the excursions may decrease in both types of hypopnoeas, Hence the use of snoring, inspiratory flattening and thoraco-abdominal paradox have been justified to diagnose obstructive hypopnoeas [6].

Non-benzodiazepine agents such as zopiclone and zolpidem may be preferable to use in the shortterm management of insomnia in OSA. Studies have documented that the use of zolpidem in doses up to $10 \mathrm{mg}$ did not impede the application of CPAP in patients with severe OSA; however, there was a reduction in sleep latency and mean arousal index [10]. The short-term use has been advocated through various studies as a safe and effective strategy for improving compliance with CPAP in patients with comorbid psychiatric disorders [11-13].

Thus, it is prudent to evaluate a patient prior to start of medical therapy for insomnia, especially in the patient subpopulation with a predisposition to obstructive sleep apnoea. Even though the exact mechanism need to be deciphered by larger studies, worsening of the underlying sleep apnoea or appearance of new central events can occur when indiscriminate or long-term prescription of benzodiazepine drugs is given to patients without complete evaluation. This can even mask the underlying obstructive sleep apnoea, thereby, delaying the diagnosis and preventing early institution of PAP therapy.

\section{Conflict of interest}

The authors declare no conflict of interest.

\section{References}

1. Dement WC, Miles LE, Carskadon MA. "White paper" on sleep and aging. J Am Geriatr Soc. 1982; 30(1): 25-50, indexed in Pubmed: 7035529.

2. Ramakrishnan K, Scheid DC. Treatment options for insomnia. Am Fam Physician. 2007; 76(4): 517-526, indexed in Pubmed: 17853625 .

3. Kaufmann CN, Spira AP, Alexander GC, et al. Trends in prescribing of sedative-hypnotic medications in the USA: 19932010. Pharmacoepidemiol Drug Saf. 2016; 25(6): 637-645, doi: 10.1002/pds.3951, indexed in Pubmed: 26711081.

4. Bailey PL, Pace NL, Ashburn MA, et al. Frequent hypoxemia and apnea after sedation with midazolam and fentanyl. Anesthesiology. 1990; 73(5): 826-830, indexed in Pubmed: 2122773.

5. Murciano D, Armengaud MH, Cramer PH, et al. Acute effects of zolpidem, triazolam and flunitrazepam on arterial blood gases and control of breathing in severe COPD. Eur Respir J. 1993; 6(5): 625-629, indexed in Pubmed: 8519370.

6. Berry RB, Budhiraja R, Gottlieb DJ, et al. American Academy of Sleep Medicine. Rules for scoring respiratory events in sleep: update of the 2007 AASM Manual for the Scoring of Sleep and Associated Events. Deliberations of the Sleep Apnea Definitions Task Force of the American Academy of Sleep Medicine. J Clin Sleep Med. 2012; 8(5): 597-619, doi: 10.5664/jcsm.2172, indexed in Pubmed: 23066376.

7. Guilleminault C. Benzodiazepines, breathing, and sleep. Am J Med. . 1990; 88(3): S25-S28, doi: 10.1016/0002-9343(90)90282-i.

8. Mason M, Cates CJ, Smith I. Effects of opioid, hypnotic and sedating medications on sleep-disordered breathing in adults with obstructive sleep apnoea. Cochrane Database Syst Rev. 2015(7): CD011090, doi: 10.1002/14651858.CD011090.pub2, indexed in Pubmed: 26171909.

9. Hanly P, Powles P. Hypnotics should never be used in patients with sleep apnea. J Psychosom Res. 1993; 37 Suppl 1: 59-65, indexed in Pubmed: 8445588.

10. Jullian-Desayes I, Revol B, Chareyre E, et al. Impact of concomitant medications on obstructive sleep apnoea. Br J Clin Pharmacol. 2017; 83(4): 688-708, doi: 10.1111/bcp.13153, indexed in Pubmed: 27735059.

11. Krakow B, Melendrez D, Lee SA, et al. Refractory insomnia and sleep-disordered breathing: a pilot study. Sleep Breath. 2004; 8(1): 15-29, doi: 10.1007/s11325-004-0015-5, indexed in Pubmed: 15026935 .

12. Takaesu Y, Inoue Y, Komada Y, et al. Effects of nasal continuous positive airway pressure on panic disorder comorbid with obstructive sleep apnea syndrome. Sleep Med. 2012; 13(2): 156-160, doi: 10.1016/j.sleep.2011.10.016, indexed in Pubmed: 22172965.

13. Lettieri CJ, Shah AA, Holley AB, et al. CPAP Promotion and Prognosis-The Army Sleep Apnea Program Trial. Effects of a short course of eszopiclone on continuous positive airway pressure adherence: a randomized trial. Ann Intern Med. 2009; 151(10): 696-702, doi: 10.7326/0003-4819-151-10-200911170-00006, indexed in Pubmed: 19920270. 